\title{
Papers to Appear in Volume 5 Number 2
}

G. H. Edwards, M. A. Etheridge and B. E. Hobbs, On the stress dependence of subgrain size

J. L. Bouchez and P. Duval, The fabric of polycrystalline ice deformed in simple shear

J. Imhof, Determination of the orientation distribution function from one pole figure

C. Esling, E. Bechler-Ferry and H. J. Bunge, Three dimensional texture analysis after Bunge and Roe: Correspondence between respective mathematical techniques 